\title{
Efficacy of Channel Estimation and Efficient use of Spectrum using Optimised Cyclic Prefix (CP) in MIMO-OFDM
}

\author{
Shovon Nandi, Narendra Nath Pathak, Arnab Nandi
}

\begin{abstract}
The performance of MIMO-OFDM network gets affected due to problems in the channel estimation which is essential for designing receivers. The issues in MIMO are due to sizable inadequate Cyclic Prefix (CP) in admired Orthogonal frequency division multiplexing (OFDM), a massive amount of detection error and channel estimation problem occurs due to which performance delay turn out. This paper proposes a solution, its implementation and analysis of results. The article uses the BAT and Modified Flower Pollination (MFP) algorithm for optimizing the CP length. Minimum CP length gets considered to achieve better throughput for the same MFP algorithm. This algorithm has been proposed to optimize $C P$ length to achieve minimum error rate of bit (BER) and PAPR-Peak to average power ratio, thereby maximizing the spectrum efficiency. For comparing the efficacy of optimized $C P$ and unoptimized $C P$, the paper examines the results obtained on studying the performance in the spectrum. The performance of BAT and MFP algorithm are compared with each other to determine the better optimization methodology for the problem.
\end{abstract}

Keywords: BAT Algorithm, Bit Error Rate (BER), Cyclic Prefix (CP), MIMO-OFDM, Modified Flower Pollination Algorithm (MFPA), PAPR

\section{INTRODUCTION}

The method of working for MIMO is multiplying the range of a tie-up applying various transmitting and receiving antennas for exploiting multipath propagation. Orthogonal Frequency Division Multiplexing is an encoding technique applied on multiple carrier frequencies. Also, the orthogonality allows great spectral efficiency with net symbol rate closer to the Nyquist rate in a comparable baseband signal [1]. In the OFDM method, a transmitter produces clock and reference signals for achieving a specific phase in carrier modulation. The receiver thereby gets the ability to recreate the reference signal, irrespective of $\mathrm{S} / \mathrm{N}$ ratio. The OFDM includes the following steps:

Revised Manuscript Received on December 30, 2019.

* Correspondence Author

Shovon Nandi*, Department of Electronics and Communication Engineering, Bengal Institute of Technology, Under Maulana Abul Kalam Azad University of Technology, Kolkata, India. Email: nandi.shovon@yahoo.in

Narendra Nath Pathak, Department of Electronics and Communication Engineering, Dr. B. C. Roy Engineering College, Durgapur, India.

Arnab Nandi, Department of Electronics and Communication Engineering, National Institute of Technology, Silchar, India.

(C) The Authors. Published by Blue Eyes Intelligence Engineering and Sciences Publication (BEIESP). This is an open access article under the CC BY-NC-ND license (http://creativecommons.org/licenses/by-nc-nd/4.0/)
- A reference signal among the AM - modulating class of digital signals with a plethora of carriers is frequency-modulated at a symbol period.

- Amplitude modulated carrier gets modulated into the primary carrier.

The frequency-modulated main carrier, the reference signal reproduced by phase-locking gets detected along with the reference signal that gets transmitted and FFT transformation for the combined carrier for demodulating the digital signal. MIMO-OFDM generally trade-off the air interface for $5 \mathrm{G}$ and $4 \mathrm{G}$ wireless broadband communication. It combines MIMO technology that improves the signal transmission capacity using various OFDM and antennas, that bifurcates the radio channel to various sub-channels that are spaced closely for providing better dependable communications with high speed scenario [2]. Research conducted during the middle of the 90's decade showed that other air interfaces at the period could also use MIMO such as Time division multiple access i.e. TDMA and Code division multiple access i.e. CDMA. MIMO-OFDM combination gets popularity in practical at high data rates. The MIMO - OFDM process acts as the base of hi-tech mobile broad-band web-work and Wireless fidelity standards since it can achieve the highest spectral efficiency. Therefore, it delivers with both highest capacity and highest data throughput. The paper [3] demonstrated that different information streams could be transmitted at the same time on a similar frequency because of signals that get transmitted through space bounce off the objects and take multiple paths to the receiver. Rayleigh proved that the MIMO processing at higher speeds mostly use OFDM modulation and transform an expeditious data channel into parallel low-speed one. The article [4] gives a complete explanation. This paper gives explanations on different MIMO-OFDM models. Shirake and Patil, 2016, reviews the co-operative OFDM system and different techniques used in the OFDM system for improving communication [5]. Sure and Bhuma, 2017, has surveyed OFDM channel estimation techniques that utilize denoising strategies [6].

\section{LITERATURE REVIEW}

The paper [7] observes that PAPR has a vital role in the MIMO-OFDM system. To defeat the issue of optimum phase factor in the partial transmit sequence (PTS) scheme, the paper combined space-time block coding (STBC) and the PTS based improved Artificial bee colony algorithm thereby increasing the complexity in the system with respect to computations. In order to reduce the complexity of an enhanced Artificial bee colony algorithm the suboptimal method gets utilized.

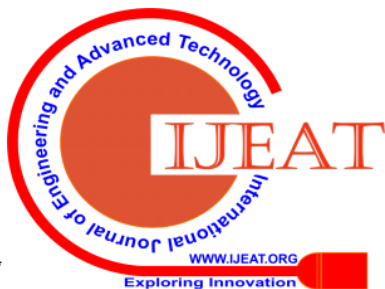




\section{Efficacy of Channel Estimation and Efficient use of Spectrum using Optimised Cyclic Prefix (CP) in MIMO-OFDM}

The paper combines PTS and STBC dependent improved ABC (Artificial Bee Colony) algorithm for reducing PAPR and BER. The BER and PAPR in the OFDM system get reduced through orthogonal initialization process and suboptimal attitude towards the ABC algorithm. Proposed hypothesis gets proved through simulation using MATLAB software.

In paper [8], the researchers had recommended a repetitive structure for estimating the channel and detecting the data for MIMO-OFDM with an inadequate $\mathrm{CP}$ and restricted quantity in pilot subcarriers. The paper proposes a multi-step channel estimation process assisted by many subcarriers. Initially, the covariance matrix of the channel and the channel path number is obtained by estimating the least squares from the channel at the subcarriers of the pilot. The paper develops a maximum likelihood viewpoint to approximating the channels of the time-domain. This paper proposes a highly performing Bidirectional M-algorithm (BDMA) for equalization based on trellis. The results from the simulation indicate that the estimated Mean square error (MSE) of the medium merges to the Cramer-Rao Bound subsequent some iterations. Also, the sufficient $\mathrm{CP}$ is achieved by the obtained BER, even if CP is much shorter than delay spread.

The article [9] described the effect of the pilot insertion and cyclic prefix methods in an OFDM which gets scrutinized on a channel of AWGN type. The paper also explains the variations in the rate of information loss and power loss along with the cyclic prefix length.

Wavelength packet transform based OFDM (WPT-OFDM) is another technology proposed in [10], investigates the technology's performance. Simulations of dual-polarization WPT-OFDM transmission are conducted in the proximity of polarization mode dispersion (PMD) and compared with traditional Fourier transform-based optical OFDM (FT-OOFDM). The outcomes prove that OFDM is touchy to PMD, acquiring 1-dB fine at 5-11ps differential group delay (DGD) for 112 GB/s dual polarization transmission.

This work analyses the non-linearity and PAPR performance of WPT-OFDM. The paper shows that Haar wavelet has improved $0.9 \mathrm{~dB}$ in non-linear power limit of launch in comparison to FT-OFDM in a traditional configuration of transmission scattering where inline scattering gets completely offset through a dispersion-compensation-fibre (DCF). In an OFDM, the reference and the clock signal is produced by the transmitter in order to give a precise phase in modulating the carrier. Therefore, the receiver can reproduce the reference signal for providing even when the SNR of the carrier is dearth of evidence. The OFDM is inclusive of the AM - Modulating steps for a collection of digital signals with a plethora of carriers, wherein the reference signal is frequency modulated at the specified period merging the carrier of the AM and then modulating the resultant signal into the main carrier. The digital signal is demodulated by the detection of the main carrier using the phase locking technique and combining it with the frequency-modulated reference signal that is reproduced [11]. For a more detailed explanation on next generation OFDM, it gets reviewed by M. Jiang and L. Hanzo [12]. The paper discusses the basics of OFDM techniques, the role of OFDM in communication technologies. Another review on OFDM is carried-out in [13]; that discussed the methodologies to increase the BER performance from architecture point. Finally, PSO gets utilized for pilot tone designs in the MIMO-OFDM system in [14], and ABC utilization is in [15].

\section{PROPOSED METHODOLOGY}

\section{A. Flowchart explanation}

The flowchart below in Fig. 1 gives the proposed methodology which aims at improving the efficiency of the spectrum through the optimization process and identifying which of the provided two methods of optimization is more CP efficient. In [16-17], we get the explanations of the nature-inspired algorithms.

\section{B. Generation of MIMO-OFDM Signals}

Paper [18] provides a detailed review of the generation of MIMO-OFDM. The review gives a comprehensive analysis of the OFDM technique and also gives an outline for various options in future work on wireless communication.

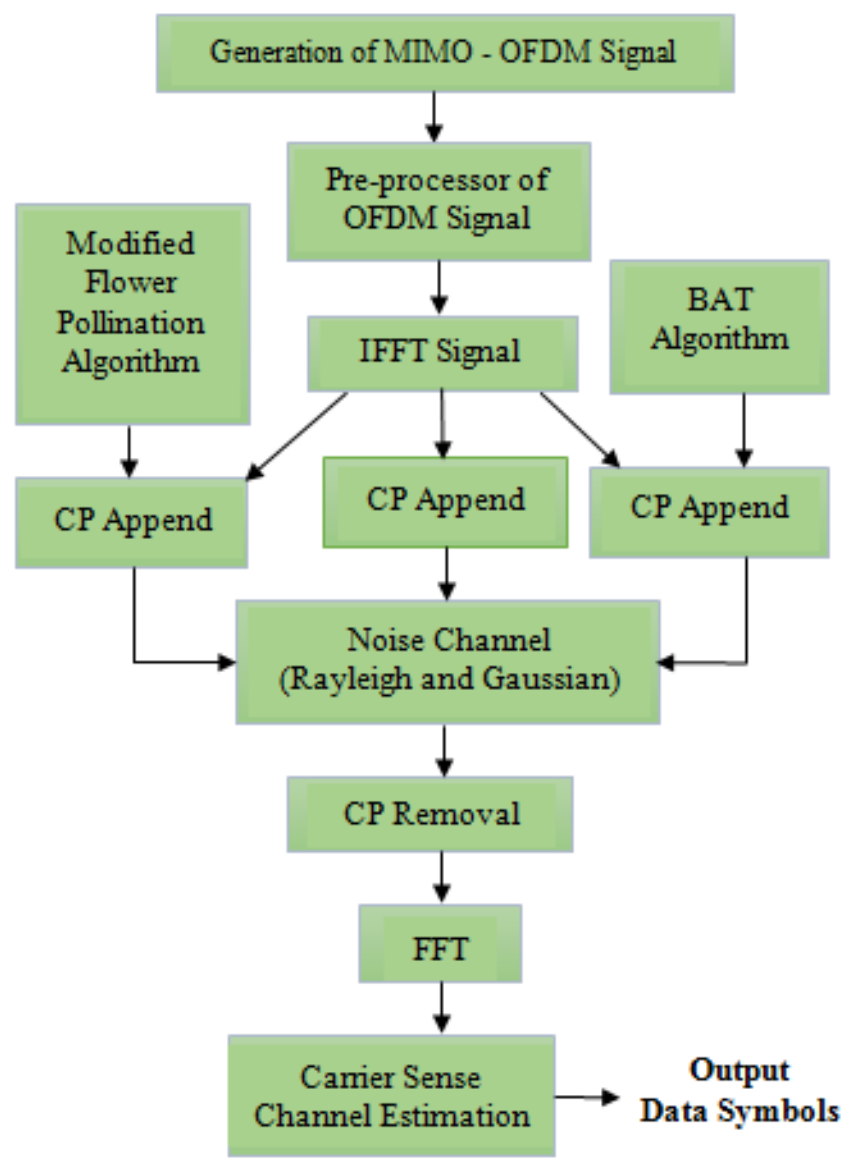

Fig. 1. Flowchart of Proposed Method.

It is far lower than that forced their ideal greatest maximum likelihood (ML) MUDs-helped partners. This article reviews the literature on the developments in MIMO-OFDM technology. 


\section{Pre-processing of OFDM signals}

Symbol mapping gets done in the pre-processing block of OFDM signals. The OFDM signals have an issue with high PAPR. For reducing the PAPR, distortionless selected mapping (SLM) was proposed, but high data processing complicatedness prevents from getting applied to the OFDM system with numerous subcarriers. The suggestion is that the OFDM amalgamated with TDM deploying minimum MSE frequency-domain equalization (MMSE-FDE) process. The paper exploits time as well as frequency dimensions of TDM/OFDM signal for improving efficacy concerning BER and PAPR. To supplement the simulation results, a SLM based scientific displaying for PAPR dispersion of TDM/OFDM is calculated. It shows that the proposed SLM can decrease the PAPR and also minimize the BER of the communicated message by means of reducing the complexity of computation. To know more about pre-processing refer [19].

\section{IFFT and FFT}

IFFT is used to modulate the signals that are to be transmitted while FFT gets used for demodulation of the received signal. The FFT is an algorithm that uses the sampling of signals for a period or space and then divided into its frequency components. These components are sinusoidal and single oscillations at peculiar frequencies each with its amplitude and phase. Fig. 1 shows the transformation and flowchart of the proposed method. The signal contains three unique dominant frequencies. The paper [20] has given a brief description of IFFT.

\section{E. CP Append}

In transmitting the OFDM symbol, ISI (Inter Symbol Interference) occurs at the receiver as a results of the delay at the channel. The guard interval gets inserted between subsequent OFDM symbols to avoid the ISI. The guard interval is planted in respect to delay spread of the channel to bypass ISI, the basics of CP are given below. interference among the symbols of OFDM. Involving multipath channels issue the orthogonality forfeit of the carriers which can be refurbished using CP.

The switching between linear convolution channel to a circular convolution channel is done by CP; thereby restoring the orthogonality of the receiver. The main drawback is that the energy is wasted in case of cyclic prefix samples.

\section{F. Modified Flower Pollination (MFP) Algorithm}

The Fig. 2 shows the flowchart for the MFP algorithm. It is a metaheuristic algorithm hinged on the pollination process of flowering plants [17]. The population gets initiated.

The population is the collection of feasible solutions for the objective function. The spectrum efficiency is to be maximized by the algorithm. Local optimisation and global optimisation are done to evaluate the best solution which is again cross-checked by again searching for the solution and updated if found a new best. If the solution is within criteria, then the solution is considered as an optimal solution if not the process gets initialized. This algorithm consists of four assumptions; interval gets nominated as cyclic prefix [21]. Enough guard

The use of zeros in the guard time can help lessen the

i. Cross pollination and Biotic properties infer as a global process of pollination with pollinators carrying pollen performing levy flights.

ii. Consider self-pollination and Abiotic as local pollination. iii. Consider constancy of the flower as the probability of reproduction can be compared to the closeness of the involved flowers.

iv. A switching probability controls the local pollination and global pollination because of the physical presence and a few more factors like local pollination and wind can affect the overall pollination activities. Presented below is the flowchart for Modified flower pollination algorithm as shown in Fig. 2.

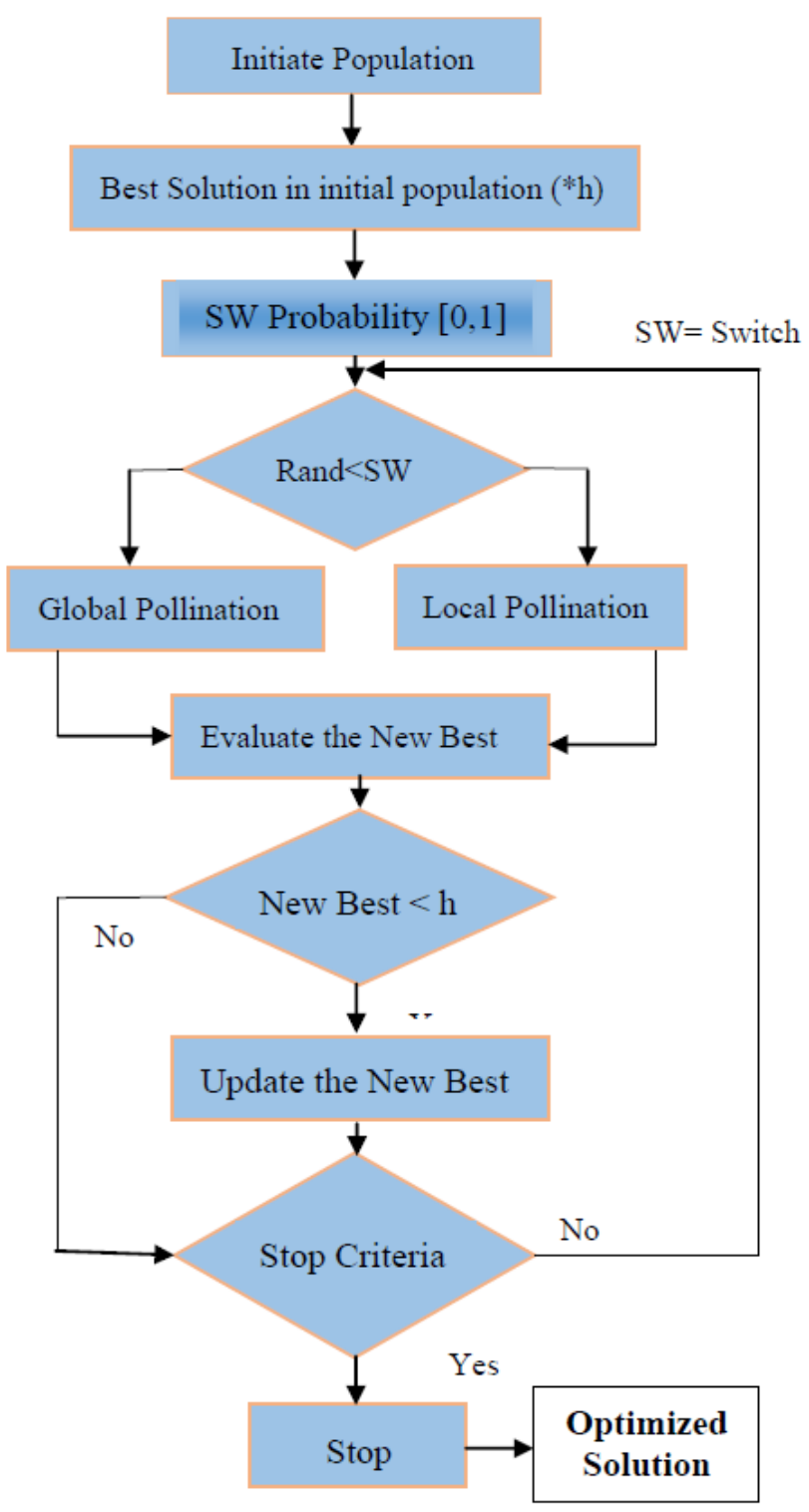

Fig. 2. Modified Flower Pollination Algorithm.

\section{G. BAT Optimisation Algorithm}

The BAT algorithm is a metaheuristic figuring, which is valuable for worldwide optimisation. The inspiration for this algorithm is the echolocation behaviour of microbat, with fluctuating rate of pulses for emission and loudness.

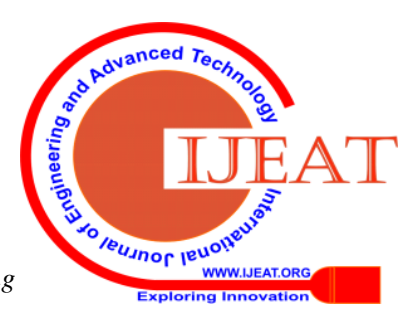




\section{Efficacy of Channel Estimation and Efficient use of Spectrum using Optimised Cyclic Prefix (CP) in MIMO-OFDM}

The first proposal of the algorithm was in [22] and first applied for engineering solutions in that article. The synopsis of the admiration of echolocation of microbats is as per the following:

a. Each bat (virtual) will arbitrarily fly with speed at position (arrangement) alongside a fluctuating wavelength or frequency and loudness.

b. For locating its prey, it varies, pulse emission rate, frequency and loudness. Search gets intensified with a nearby arbitrary walk. The process of selecting the finest continues until the meeting of specific stop criteria happens. The process primarily utilizes a frequency-tuning method for controlling the dynamic behaviour of swarm bats. The careful control for balance among exploitation and exploration is carried out by tuning the parameters that are dependent on the tuning algorithm. Yang, 2010, gives a brief introduction about meta-heuristic algorithms including BAT algorithm [16] and a brief review on the nature-inspired algorithm is carried out in [23]. Presented below in Fig. 3 is the flowchart for the BAT Algorithm.

Initiate BAT population with Position \& Velocity

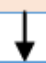

Define Pulse, Frequency and Loudness of BATS

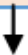

Generate new BAT position by adjusting

Velocity, Frequency and Location

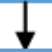

Move BAT location locality with Random Walk

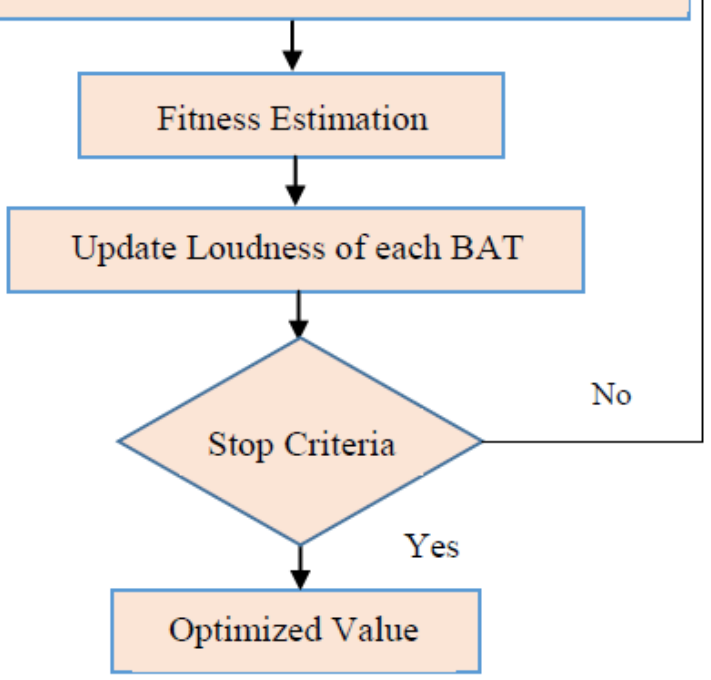

Fig. 3. Flowchart for BAT Algorithm.

\section{H. Communication Channel}

Additive substance White Gaussian Noise (AWGN) is a channel model where just harm in correspondence channel is a direct option of repetitive white noise or wide band with constant spectral density and Gaussian appropriation of amplitude. This model neglects fading, interference, non-linearity, dispersion or frequency selectivity. AWGN is common for all transmission channels. It is a random noise

characterized by the wide range in frequencies regarding a signal in the communication channel.

Rayleigh distribution portrays the time-differing nature of the bundle which is gotten from the flat fading signal or envelope of an individual multipath part. The total of the envelope of the given two quadrature Gaussian commotion signal complies with a Rayleigh dissemination. The weakening of signal causes the main component to escape getting noticed among the multipath components, originating Rayleigh model.

\section{CP Removal}

The previously added symbols to the signal have got removed in the CP removal process which gets explained in Fig. 4. The CP removal needs while demodulating, for maintaining the continuous flow of data in the receiver. The $\mathrm{CP}$ length is reduced to improve performance and for easier removal, discussed in [24].

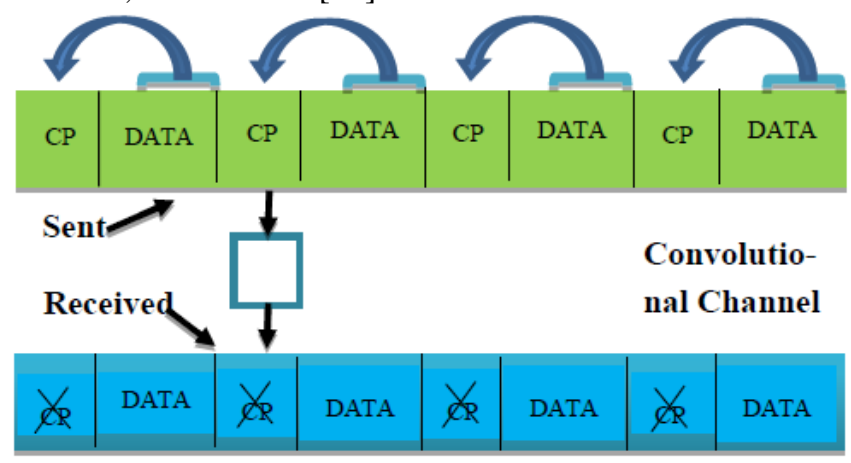

Fig. 4. CP Removal from Data.

\section{J. Carrier Sense Channel Estimation}

By activating all sources simultaneously, estimation of MIMO channel takes place. The receiver measures the cumulative response consisting of convolutions chose randomly between different sets of source signals and channel reactions. The main aim is the reduction of the time required for estimating for minimizing it to recovers the channel response [25].

\section{K. Operation}

The input data symbols get mapped after combined with pilot tones inserted using an IFFT. The modulated data gets transmitted through sending end antennas. This paper explains a general operation of the MIMO-OFDM system and the receiver uses a demodulator. FFT is used here and the signal gets transmitted to carrier sense channel estimation from which decoding of data symbols gets done.

The generation of the MIMO-OFDM signal took place and shown in this context. Pre-processing of OFDM signals getting modulated using IFFT gets carried out. The modulated signal relies on the quantity of bits per symbol, size of signal constellation, no. of samples per symbol and no. of transmitter antennas. The paper performs three different operations for CP. Optimisation of appending cyclic prefix is carried out by the Modified flower pollination algorithm.

Similarly, the CP is optimized using BAT algorithm also for getting a comparative view.

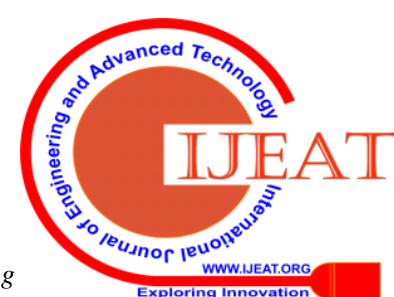


To get the CP-removed, the results of the algorithm get transmitted through noise channel, and the $\mathrm{CP}$ gets removed before getting transformed using the FFT technique. The transmission of the carrier sense channel estimation detects the pilot signal with the actual data signal, and the quality of the signal is observed to determine the quality of the actual data signal. Input signal given is a discrete signal as shown in Fig. 5, which gets modulated with the OFDM signal, shown in Fig. 6, as the discrete signals require a carrier wave for signal transmission. The finally produced modulated signal is shown in Fig. 7.

IFFT (Inverse Fast Fourier Transform): The time space portrayal of OFDM is comprised of various orthogonal sinusoidal signals which is IFFT. For converting frequency domain to the time domain, the transmitter requires a mathematical operation of IFFT.

Cyclic Prefix (CP): The term refers to prefixing of a symbol with a repetition of the end. The receiver is configured to discard CP samples, but CP has two objectives. i. A guard interval gets provided for eliminating intersymbol interference from the previous symbol.

ii. The tail of the symbol repeats so that displaying of the linear convolution of a frequency specific multipath channel can be the circular standard. This, in turn, may transform into the frequency domain through DFT or FFT.

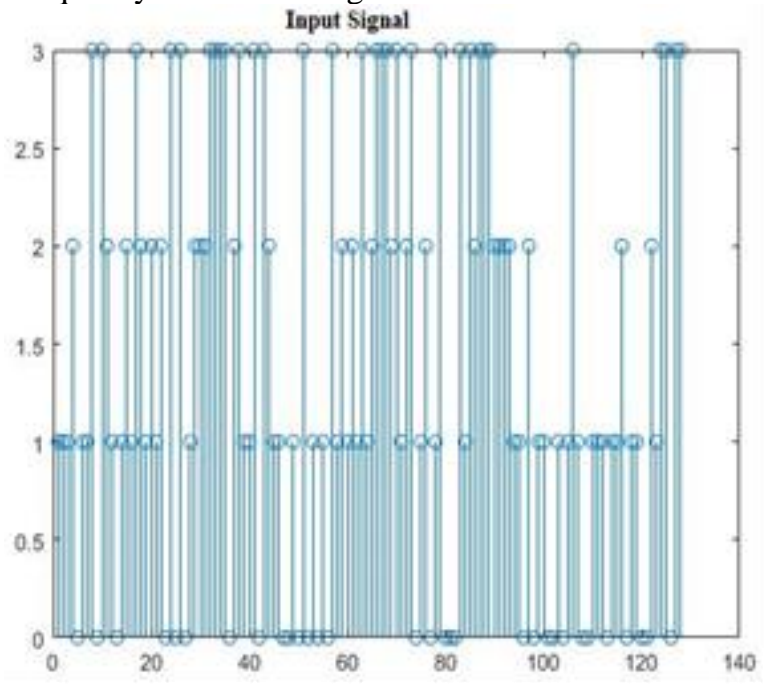

Fig. 5. Input Signal.

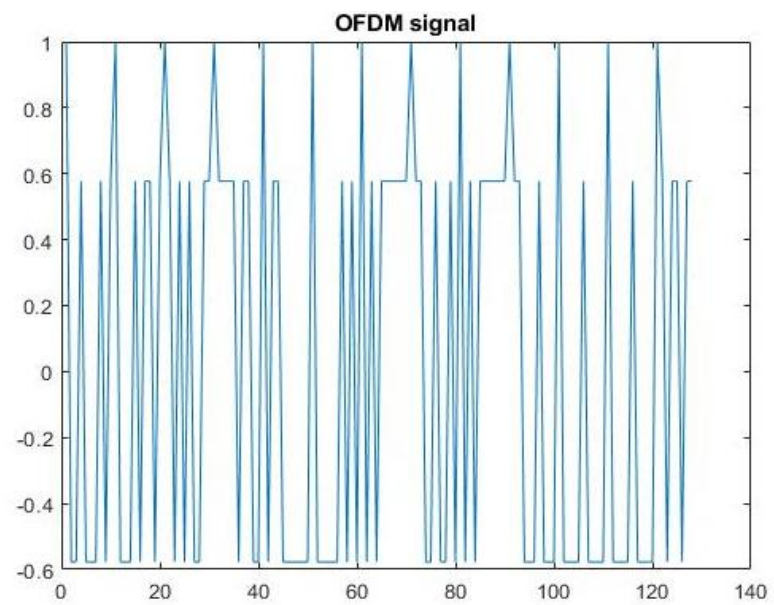

Fig. 6. OFDM Signal.

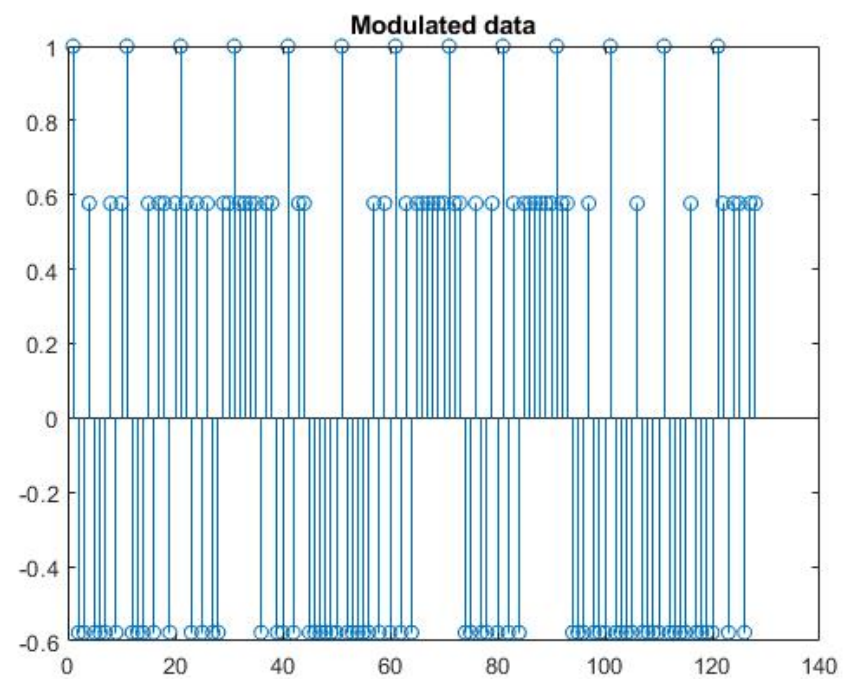

Fig. 7. Modulated Signal.

CP Length Optimisation using Optimisation Algorithm: This project has considered three CP lengths, firstly the unoptimised CP length has been considered than optimized $\mathrm{CP}$, has been inserted using BAT and MFP algorithms respectively.

Modulator (QAM): The considered system uses ' $\mathrm{M}$ ' order QAM modulation. Having generated the data symbols in the column vector, use the QAM mod function for applying M-QAM modulation for both binary and gray coded bit-to-symbol mappings.

\subsection{Formulation: Objective Function:}

The actual formula is followed here to achieve the following targets;

i. Minimize PAPR and ii. Minimize BER

The peak to average power ratio (PAPR) is given by,

$$
\text { PAPR }_{\mathrm{dB}}=10 \log _{10} \frac{\| \mathrm{x}_{\text {peak }}^{2} \rrbracket}{\mathrm{x}_{\text {Inus }}^{2}}=\mathrm{C}_{\mathrm{dB}} \text {; }
$$

For probability $\mathrm{X}<=\mathrm{x}$ and the range of $\mathrm{x}$ is $0<\mathrm{x}<1$.

The Bit Error Rate (BER) is given by

$$
\text { BER }=\frac{\text { No.of Errors }}{\text { Total no.of bits sent }} \text {; }
$$

The PAPR and BER are to be computed and optimised using the proposed algorithm discussed in this paper. 


\section{Efficacy of Channel Estimation and Efficient use of Spectrum using Optimised Cyclic Prefix (CP) in MIMO-OFDM}

\section{RESULTS AND ANALYSIS}

The PAPR for CP gets plotted without optimizing the solution (Channel State Estimation - CSE). The unoptimised solution generally is not the best possible solution. There is a provision for better solutions is optimized (CSE BAT). The probability reaches 0.5 at 9 approximately. The PAPR for CP gets plotted by optimizing the solution using BAT algorithm. The optimized solution may or may not be the best possible solution. Better solutions possibly can be provided when optimized by another algorithm. Here the probability reaches 0.5 at 8 approximate. On the other hand, our proposed modified flower pollination algorithm provides better possible solution (CSE BAT).

Here the probability reaches 0.5 at 7 approximately when optimized by MFP, as shown in Fig. 8.

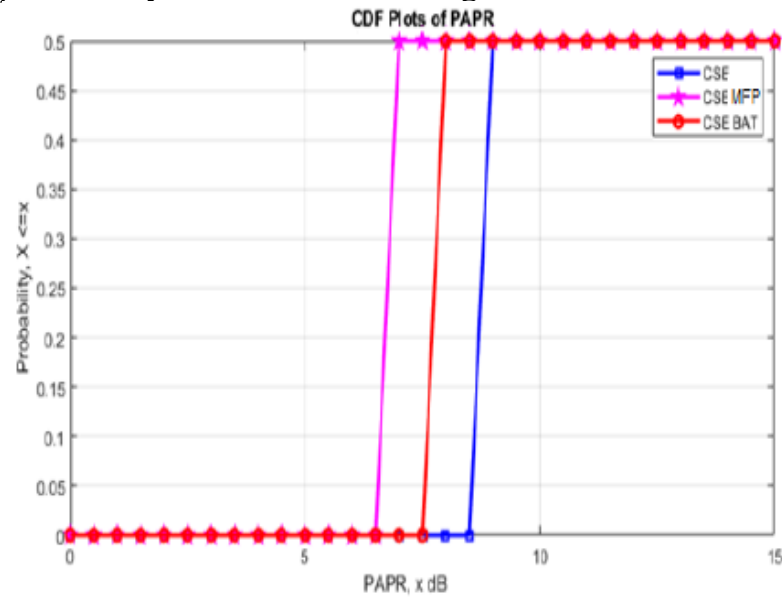

Fig. 8. PAPR for Optimized (MFP, BAT) and Unoptimised CP.

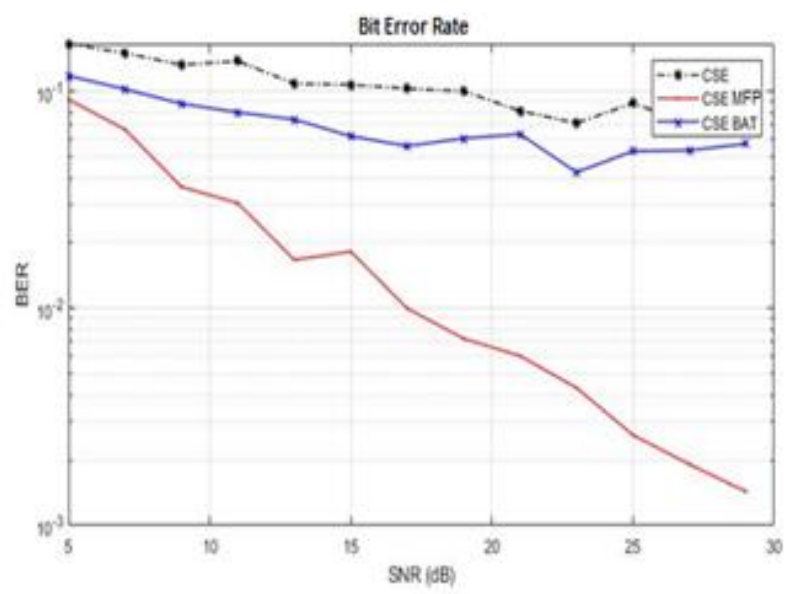

Fig. 9. BER for optimized (MFP, BAT) and unoptimised CP.

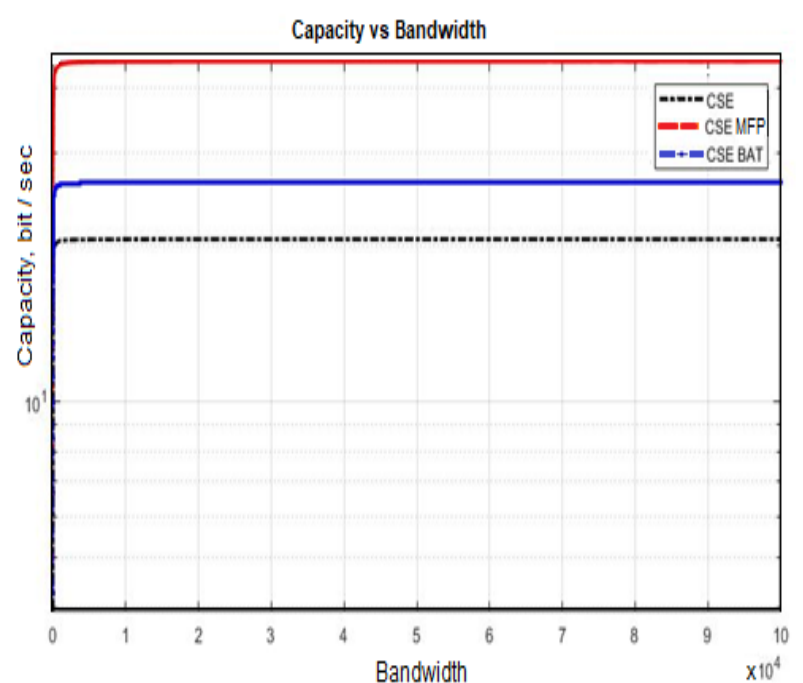

Fig. 10. Bandwidth for optimized (MFP, BAT) and unoptimised CP.

The results obtained from Cumulative Distribution Function CDF plots for PAPR when optimisation happens, it shows that as the PAPR increases, the probability of $\mathrm{X}(<=\mathrm{x})$ also increases. The probability rises at lower decibels for BAT algorithm compared to the unoptimized solution. MFP algorithm performance is better than the BAT algorithm as it reaches the highest probability at the lower decibel level of the signal.

The bit error rate gets compared for unoptimised (CSE), BAT algorithm (CSE BAT) and MFP algorithm (CSE MFP) which shows that bit error rate reduces for all the algorithm for higher $\mathrm{S} / \mathrm{N}$ ratio. The detailed comparison is shown in Fig. 9. The BER decreases faster for MFP compared to BAT algorithm which gets deduced on examining the BER performance.

With the rapid transformation of the communication demand into the efficient digital podium, the aim is to add sense of optimization to achieve efficient bandwidth in Fig. 10. It believes that to mitigate the demand of the multi-media data transmission through communication channel can only be possible by the efficient bandwidth usage. Here also the MFP algorithm provides greater bandwidth as compared with the unoptimized and BAT algorithm. Hence it can be deduced that MFP algorithm is performing better than BAT algorithm and which in turn is responsible for betterment of spectrum efficiency.

\section{ConClusion}

On observing various parameters in the above experiment, results on investigating demonstrates that the exhibition of CP with optimisation is better than CP without optimisation. Performance of CP, when optimized using MFP algorithm, is better than CP performance when optimized through BAT algorithm. BER drastically reduces for MFP algorithm since 'Signal to Noise Ratio' is better for MFP compared to BAT and unoptimised solutions; it also results in better spectrum efficiency. 
The possible future work in this field is identifying better optimisation algorithms for improving the performance of MIMO-OFDM. The MFP algorithm can be replaced by a hybrid flower pollination algorithm to test the outcome for comparison. Moreover, there are developments of a Single-User (SU) and Multi-User (MU) MIMO. The future work can perform a comparison of both technologies. Moreover, there are developments of a Single-User (SU) and Multi-User (MU) MIMO. The future work can perform a comparison of both technologies.

\section{REFERENCES}

1. H. Rohling, OFDM: Concepts for Future Communication Systems, Signals and Communication Technology, Springer-Verlag Berlin: Heidel-berg, 2011.

2. Anuja Shirake \& S. V. Patil, "A Review on Cooperative OFDM System for Wireless Communications”, International Journal of Scientific Development \& Research, 4(6), 126-130, 2016.

3. Yong Soo Cho, Jaekwon Kim, Won Y. Yang, Chung G. Kang, MIMO-OFDM Wireless Communications with MATLAB, John Wiley and Sons, ISBN: 978-0-470-82562-4, 2010.

4. D. Kumutha, \& N. A. Prabha, "Hybrid STBC-PTS with enhanced artificial bee colony algorithm for PAPR reduction in MIMO-OFDM system", Journal of Ambient Intelligence and Humanized Computing, 1-17, 2017.

5. Tomoki Saeki, Orthogonal frequency division multiplexing. US Patent, US5956318A, 1996.

6. P. Sure \& C. M. Bhuma, "A survey on OFDM channel estimation techniques based on denoising strategies", Engineering Science and Technology, An International Journal, 20(2), 629-636, 2017.

7. T. Pham, T. Le-Ngoc, G. K. Woodward \& P. A. Martin, "Channel estimation and data detection for insufficient cyclic prefix MIMO-OFDM", IEEE Transactions on Vehicular Technology, 66(6), 4756-4768, 2017.

8. A. Li, W. Shieh \& R. S. Tucker, "Wavelet packet transform-based OFDM for optical communications", Journal of Lightwave Technology, 28(24), 3519-3528, 2010.

9. M. Bhardwaj, A. Gangwar \& D. Soni, "A review on OFDM: concept, scope \& its applications", IOSR Journal of Mechanical and Civil Engineering (IOSRJMCE), 1(1), 07-11, 2012.

10. C. Manicandan, P. Neelamegam, \& E. Divya, "OFDM techniques for MIMO-OFDM system: A Review”, Indian Journal of science and Technology, 8(22), 15, 1-4, 2015.

11. S. Shashikant, \& D. Dhawan, "Cyclic prefix optimization of OFDM system”, IOSR J. Electron. Commun. Eng. (IOSR-JECE), 9(3), 79-82, 2014.

12. M. Jiang \& L. Hanzo, "Multiuser MIMO-OFDM for next-generation wireless systems", 95(7), 1430-1469, 2007.

13. D. Skraparlis \& J. D. Kanellopoulos, "Design of pre-processing algorithms for efficient MIMO-OFDM receiver architectures", 3(1), 37-47, 2010.

14. X. S. Yang, \& A. H. Gandomi, "Bat algorithm: a novel approach for global engineering optimization, Engineering Computations", 29(5), 464-483, 2012

15. X. G. Xia, "Precoded and vector OFDM robust to channel spectral nulls and with reduced cyclic prefix length in single transmit antenna systems", IEEE Transactions on Communications, 49(8), 1363-1374, 2001.

16. M. Morelli \& U. Mengali, "Carrier-frequency estimation for transmissions over selective channels", IEEE Transactions on Communications, 48(9), 1580-1589, 2000.

17. D. Meenakshi, S. Prabha \& N. R. Raajan, "Compare the performance analysis for FFT based MIMO-OFDM with DWT based MIMO-OFDM", International Conference on Emerging Trends in Computing, Communication and Nanotechnology (ICE-CCN), IEEE, 441-445.

18. E. Nabil, "A modified flower pollination algorithm for global optimization", Expert Systems with Applications, 57, 192-203, 2016.

19. X. S. Yang, "A new metaheuristic bat-inspired algorithm”, In Nature inspired cooperative strategies for optimization (NICSO 2010), Springer, Berlin, Heidelberg, 65-74, 2010.

20. X. S. Yang, "Nature-inspired-metaheuristic algorithms", Elsevier, 2014.

21. S. Nandi, A. Nandi, N. N. Pathak \& M. Sarkar, "Performance analysis of Cyclic Prefix OFDM using Adaptive Modulation Techniques", IJEECS, ISSN 2348-117, 6(8), 214-220, 2017.
22. X. Rodet \& P. Depalle, "Spectral envelopes and inverse FFT synthesis", In Audio Engineering Society Convention 93, Audio Engineering Society, 1992.

23. R. S. Parpinelli \& H. S. Lopes, "New inspirations in swarm intelligence: a survey", International Journal of Bio-Inspired Computation, 3(1), 1-16, 2011.

24. S. Nandi, A. Nandi \& N. N. Pathak, "Performance analysis of Alamouti STBC MIMO OFDM for different Transceiver System", IEEE organized International Conference on Intelligent Sustainable System (ICISS), 2017.

25. Jia. Meng, Yin. Wotai, Li. Yingying, Nam. Tuan. Nguyen \& Han. Zhu, "Compressive Sensing based High Resolution Channel Estimation for OFDM System", IEEE Journal of Selected Topics in Signal Processing, 2012

\section{AUTHORS PROFILE}

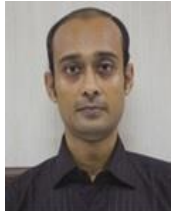

Shovon Nandi, received $M$. Tech degree from Calcutta University, India is now working as an Assistant Professor at Bengal Institute of Technology, Kolkata, under Maulana Abul Kalam Azad University of Technology (MAKAUT), affiliated by AICTE, India. His research interests include multicarrier techniques such as OFDM, MC-CDMA and MIMO systems and wireless sensor networks.

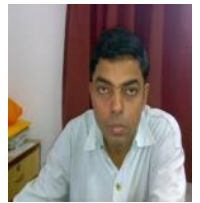

Narendra Nath Pathak, received Ph.D. degree from National Institute of Technology, Durgapur, India, is attached with Dr. B. C. Roy Engineering College as a Professor \& Head of Electronics \& Communication Engineering Department. His research interests include antennas and propagation, MIMO OFDM system, channel estimation and wireless sensor

networks.

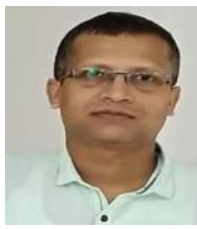

Arnab Nandi, received Ph.D. from National Institute of Technology, Durgapur, India, is attached with National Institute of Technology, Silchar, India, as an Assistant Professor of Electronics \& Communication Engineering Department. His research interests include wireless sensor networks, relay networks, cognitive radio and antenna. 